\title{
Selection of Optimal Beam Forming Algorithm for Mobile Ad Hoc Networks
}

\author{
Mohammed Tarique \\ Department of Electrical Engineering Ajman University of Science and Technology, Fujairah, United Arab Emirates \\ Email: m.tarique@ajman.ac.ae
}

How to cite this paper: Tarique, M. (2017) Selection of Optimal Beam Forming Algorithm for Mobile Ad Hoc Networks. Wireless Engineering and Technology, 8, 20-36. http://dx.doi.org/10.4236/wet.2017.81002

Received: December 19, 2016

Accepted: January 21, 2017

Published: January 24, 2017

Copyright $\odot 2017$ by author and Scientific Research Publishing Inc. This work is licensed under the Creative Commons Attribution International License (CC BY 4.0).

http://creativecommons.org/licenses/by/4.0/

\section{(c) (i) Open Access}

\begin{abstract}
Ad hoc networks have drawn considerable attentions of researchers for the last few years. Various applications of ad hoc networks have been reported in the literatures including disaster management, battle field, environmental management, healthcare, and smart grid. Ad hoc networks have some limitations namely short operating life, unreliability, scalability, delay, high interference, and scarce resources. In order to overcome these limitations, numerous researches have been carried out. Smart antenna integration is one of them. It has been shown in the literatures that smart antenna can improve network's capacity, increase network lifetime, reduce delay, and improve scalability by directing antenna radiation pattern in a desired direction. But, producing a desired antenna radiation pattern is not a simple task for resource constraint ad hoc networks. A careful selection of beam forming algorithm is required. In this paper we show that smart antenna system, consisting of circular microstrip antennas and arranged in a linear arrangement, is the most suitable choice for ad hoc network. We compare a number of smart antenna algorithms in this paper under different noisy conditions. We show that the Least Square Constant Modulus (LSCM) and Least Constant Modulus (LCM) algorithms outperform other algorithms in terms of directivity and minimized side lobes.
\end{abstract}

\section{Keywords}

Ad Hoc Networks, Microstrip, Antenna Array, Smart Antenna, Beam Forming, DSP

\section{Introduction}

Recent developments in electronic devices like laptop computers, personal digital assistant (PDA), pagers, and cellular phones have become portable and "smart" now. It is very common that users roam with these devices at any place 
and at any time. Hence, there is need for a network that users can connect with on a temporary basis. Ad hoc network is considered a suitable solution for this kind of temporary network. Ad hoc network consists of a number of mobile nodes. It is self-organizing and self-configuring. No network infrastructure is required to set up this type of network. Based on these attractive features many applications of ad hoc network have already been introduced. Some of these applications include crisis management, search and recuse, telemedicine, telegeoprocessing, process control, personal communication, virtual navigation, education, and security [1].

Ad hoc network has limitations too. It has dynamic topology. Mobile nodes join and leave the network at any time. Hence, route "breakage" is a very frequent phenomenon in ad hoc network. Other limitations include high packet loss, unreliability, high interference and noise, and limited range. Like other wireless systems wireless medium is shared by a group of mobile nodes. Moreover, mobile nodes communicate with each other in a multi-hop fashion. Hence, ad hoc network can support only a limited bandwidth to an individual user. In order to improve bandwidth many researches have been carried out for the last decade. Smart antenna integration is one of them.

Smart antenna enables mobile node to radiate power in a specified direction. It also helps mobile nodes to reduce interference level in a network by steering radiation's nulls toward the sources of interference. Hence, a number of simultaneous transmissions can occur at the same time as shown in Figure 1. Smart antenna also allows a mobile node to adjust its transmission power to a required minimum level and hence improves the network life-time. There are other advantages of using smart antenna in ad hoc networks. These advantages have been comprehensively studied in the literatures [2]-[12].

In this paper we investigate effective and efficient smart antenna design strategy for mobile ad hoc networks. We consider two different antenna elements in this investigation namely circular microstrip antenna and rectangular microstrip antenna. We compare the performances of these two antennas in order to select a suitable antenna element for smart antenna design. We also consider three different antenna arrangements namely linear, planar, and circular. In addition,

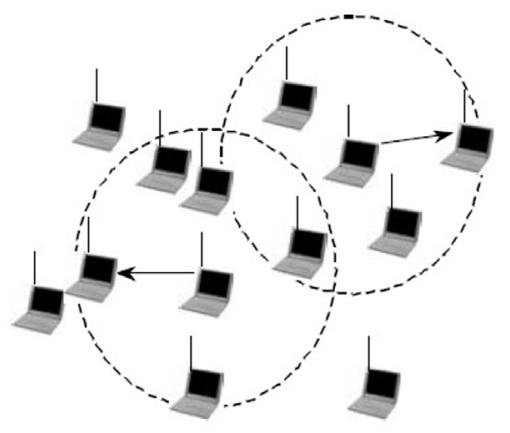

(a) Low Througput with Omnidirectional Antenna

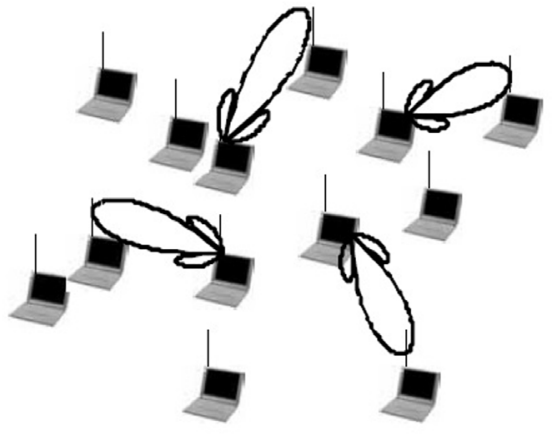

(b) High Throughput with Smart Antenna System

Figure 1. Capacity improvement of ad hoc network with smart antenna [2]. 
we investigate a number of smart antenna algorithms in order to select an optimum algorithm for mobile ad hoc networks. The rest of the paper is organized as follows. Some related works have been presented in Section 2. A brief introduction to microstrip antenna has been presented in Section 3. The performances of rectangular and circular microstrip antennas have been investigated and compared in the same section. The smart antenna design algorithms for $\mathrm{Ad}$ hoc network have been presented in Section 4. This paper has been concluded with Section 5.

\section{Related Works}

Numerous research activities have been done so far in order to improve the bandwidth of ad hoc networks. Most of the researches have been investigated based on the assumption that mobile nodes are equipped with omnidirectional antenna. With omnidirectional antenna mobile nodes radiate signal equally in all surrounding area and all mobile nodes within the transmission range receive that signal. Due to the omnidirectional radiation and imposed medium access control (MAC) algorithm the neighboring nodes remain standby during their transmission to avoid packet collision. Hence, network throughput is reduced. In order to increase network throughput smart antenna integration has been suggested for ad hoc network [2]-[11].

One of the earliest works on smart antenna application in ad hoc network has been presented in [2]. The interaction and integration of critical components of smart antenna have been addressed in this paper. The authors have shown that the performance of an ad hoc network highly depends on the adaptive beam forming algorithm and signal processing algorithm. The authors focused on the antenna pattern, training sequences, direction of arrival algorithm, and the antenna coupling effects.

In [3] the authors suggest smart antenna for wireless ad hoc networks instead of omnidirectional antennas. The authors claim that smart antenna at each mobile node facilitates simultaneous transmission of packets and hence improves the network throughput. The authors show via simulations that it is preferable to use the beamforming technique over the switched beam technique in order to obtain a high throughput.

A novel MAC protocol based on directional antenna has been presented in [4]. By using this protocol, source and destination nodes determine each other's directions. The source node sends data packets in the given direction. Hence, the interference level is reduced and network throughput is improved. The author compared the performances of the network for two cases-using omnidirectional antenna and using directional antenna. The simulation experiments presented in [4] show that the proposed protocol, with 4 directional antennas per node, increases the average throughput of the network by 2 to 3 times.

Directional Virtual Carrier Sensing (DVCS) protocol for mobile ad hoc networks has been presented in [5]. This protocol has been designed based on directional antenna. This protocol uses information on AOA (Angle of Arrival) 
and antenna gain for the adaptation of antenna pattern. The simulation results show that DVCS protocol can improve the network capacity by a factor of 3 to 4 for a small ad hoc network consisting of 100 nodes compared to omni-directional antenna based ad hoc network of the same size.

In [6] the authors suggest that directional antennas to be used for ad hoc networks instead of omni-directional antenna. They also suggest that existing MAC protocols need to be modified in order to take the advantages of directional antennas. Current MAC protocols, including popularly used IEEE 802.11 standard, have been designed for omnidirectional antenna. The authors have presented a new MAC protocol that is suitable for ad hoc networks based on directional antennas.

A distributed receiver-oriented multiple access (ROMA) channel access scheduling protocol has been presented in [7]. The ROMA protocol uses directional antennas to form multiple directional radiation pattern to ensure several simultaneous communications. The ROMA protocol activates a number of links in a time slot. The results presented in [7] show that a significant improvement on the network throughput can be achieved by using the ROMA protocol.

Capacity of ad hoc networks based on directional antenna has been formulated in [8]. The authors argued that directional antennas can support a number of simultaneous connections. But, there is a limit on such type of concurrent communications because of the mutual interference. Hence, there is a bound on the amount of capacity improvement. The network capacity bound depends on the specific antenna type and higher layer protocols. In [8] the authors calculate interference based capacity bounds for a generic antenna model and a real-world antenna model.

In a similar work [9] the authors have used beamforming antennas to increase the transmission range and also to reduce the interference level in ad hoc networks. The authors have considered a number of enhancements including "aggressive" and "conservative" channel access mechanisms based on beamforming antennas, link power control, and directional neighbor discovery. Two approaches of beam forming algorithms have been used namely steered and switched beams. The results show that beamforming improves network performance by $28 \%-118 \%$ depending upon the node density. The results also show that network delay can also be reduced by a factor of 28 by using appropriate beamforming algorithm.

It is shown in [10] that directional antennas not only increase the network capacity but also increase network life. The authors present an energy-efficient routing and scheduling algorithm in [10]. The algorithm chooses the shortest cost path to save energy. Based on the network topology information each mobile node schedules transmission so that the total time a packet takes to travel is minimized. The proposed scheduling algorithm is distributed and adaptive fashion. The simulation results show that the proposed protocol can save energy by $45 \%$ and hence can maximize the network life. 
Another energy saving MAC protocol for mobile ad hoc networks, utilizing directional antennas, has been presented in [11]. It is shown that directional antenna can help to achieve higher directivity and hence to achieve higher antenna gain. Thus, mobile node can reduce transmission power to a minimum level and can save energy. The simulation results presented in [11] show that the proposed MAC protocol not only saves energy but also maximizes network throughput.

Most of these above mentioned works focus on the performance improvement of ad hoc network by directional antenna. In this paper a design strategy for smart antenna integration in ad hoc networks has been presented. Microstrip antenna has been used as the antenna element in smart antenna design. In this work two different microstrip antennas have been considered namely rectangular microstrip and circular microstrip. In this investigation the performances of these two types of antennas have been compared. Three different types of antenna array namely linear, planar, and circular have been investigated in this work. It has also been shown that the planar and circular antenna arrays require extensive signal processing and hence they are not suitable for resource constraint ad hoc networks. In this work we also investigate a number of smart antenna algorithms namely Least Mean Square (LMS), Sample Matrix Inversion (SMI), Recursive Least Square (RLS), Constant Modulus (CM), Least Square Constant Modulus (LSCM), Conjugate Gradient Method (CGM), and Spreading Sequence Array Weights (SSAW). Based on this investigation we recommend a suitable algorithm for smart antenna for mobile ad hoc networks. None of the previously mentioned related works have addressed these issues.

\section{Microstrip Antennas}

Microstrip antennas have been widely used in wireless communication, mobile radio, spacecraft, missiles, aircraft and satellite since 1970. These wide spread applications of microstrip antennas became possible because of its low cost, small size, and easy installation. The most attractive feature of microstrip antennas is that they can be easily integrated and fabricated onto anelectronic circuit board. Microstrip antenna can be found in different shapes including rectangular, circular, square elliptical, dipole, ring, and triangular. Among these shapes rectangular and circular shapes have become popular in antenna array design.

A rectangular microstrip antenna is shown in Figure 2. It consists of a very thin metallic strip placed on dielectric substrate. The thickness of the microstrip is usually less than free space wavelength (i.e., $\lambda_{0}=c / f$ ) and the substrate thickness varies between $0.003 \lambda_{0}$ and $0.05 \lambda_{0}$. The rectangular strip is placed on the substrate in a way so that the maximum antenna radiation occurs along the direction that is normal to the microstrip. The length of the microstrip, $L$ varies between $1 / 3 \lambda_{0}$ to $1 / 2 \lambda_{0}$. A number of dielectric substrates, with the dielectric constant, $\epsilon_{r}$ from 2.2 to 12 , are used in microstrip antenna.

The expression of the fields radiated from rectangular microstrip antenna can be found in [13]. The normalized radiation field for the E-plane $\left(\theta=90^{\circ}, 0 \leq \varphi \leq\right.$ 


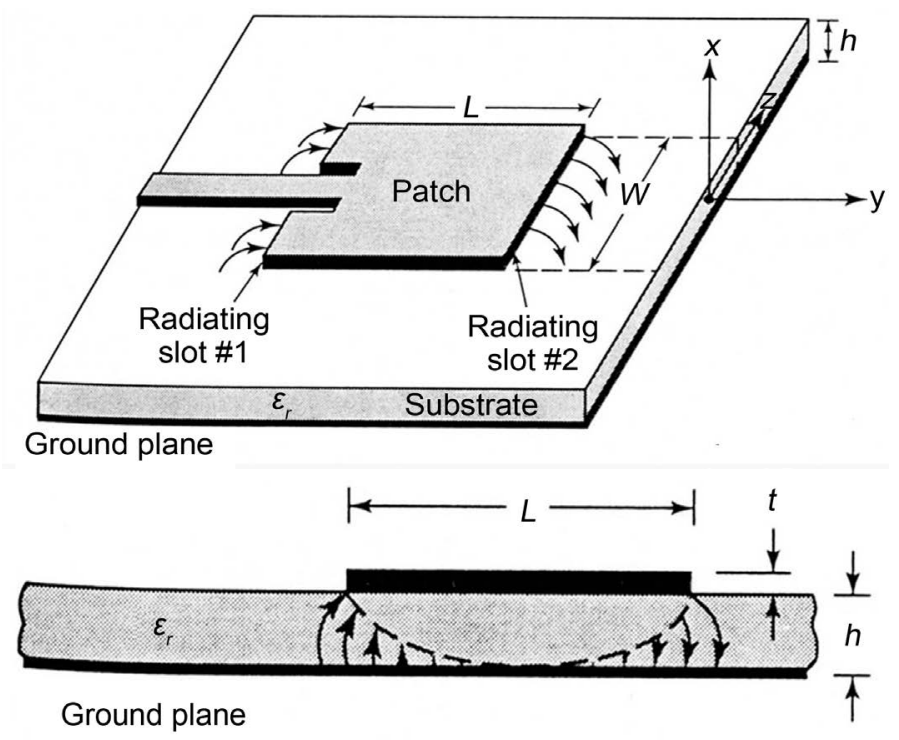

Figure 2. Rectangular microstrip antenna.

$90^{\circ}$, and $270^{\circ} \leq \varphi \leq 360^{\circ}$ ) is given by

$$
E_{\theta}=\frac{\sin c\left\{\cos \phi \frac{h k_{0}}{2} \cos (\sin \phi) \frac{k_{0} L_{e}}{2}\right\}}{\sin c\left(\frac{h k_{0}}{2 \pi}\right)}
$$

The normalized radiation field for the H-pane $\left(\varphi=0^{0}, 0^{0} \leq \theta \leq 180^{\circ}\right)$ is given by

$$
E_{\phi}=\frac{\sin \theta \sin c\left(\sin \theta \frac{h k_{0}}{2 \pi}\right) \sin c\left(\cos \theta \frac{W k_{0}}{2 \pi}\right)}{\sin c\left(\frac{h k_{0}}{2 \pi}\right)}
$$

where $L_{e}=$ effective length, and $k_{0}=2 \pi / \lambda_{0}$. The microstrip antenna is feed by a microstrip transmission line as shown in Figure 3. The input impedance of this transmission line can be varied by adjusting the length of $y_{0}$. In order to plot the field patterns, we use the MATLAB program provided in [13]. The plots for the fields in E-plane and H-plane are shown in Figure 4. The other design parameters used in producing the field pattern have been listed in Table 1 . The other values produced by the program are as follows: effective length $L_{e}=1.0685 \mathrm{~cm}$, E-plane Half Power Beam Width $(\mathrm{HPBW})=88^{\circ}, \mathrm{H}$-plane Half-Power Beam Width $(\mathrm{HPBW})=76^{\circ}$, directivity, $D_{0}=5.3514$, resonant input resistance at feed point $R_{\text {in }}=228 \Omega$.

A circular microstrip antenna is shown in Figure 5 . The construction of a circular microstrip antenna is similar to that of a rectangular microstrip antenna. Only the shape of the metallic strip is circular rather than rectangular.

The normalized radiation field for a circular microstrip antenna in the E-plane $\left(\theta=90^{\circ}, 0 \leq \varphi \leq 90^{\circ}\right.$, and $\left.270^{\circ} \leq \varphi \leq 360^{\circ}\right)$ is given by

$$
E_{\theta}=J_{0}\left[\sin (\theta)\left(k_{0} a_{e}\right)\right]-J_{2}\left[\sin (\theta)\left(k_{0} a_{e}\right)\right]
$$




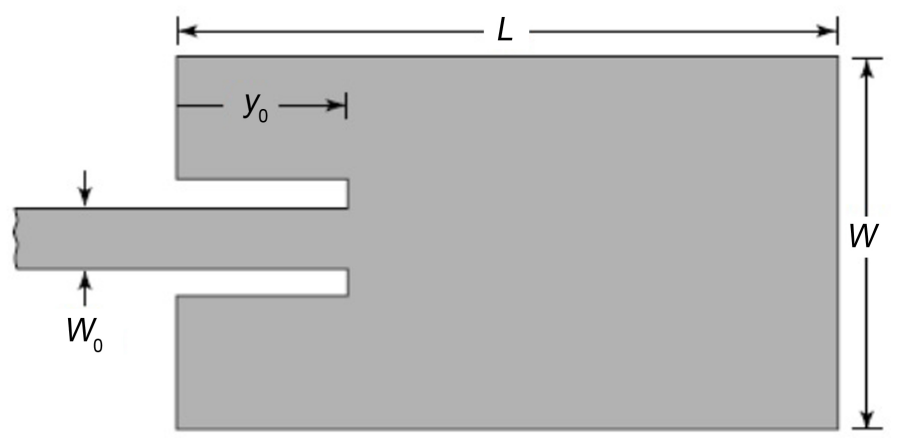

Figure 3. The feed mechanism of microstrip antenna.

E- and H-plane Patterns of Rectangular Microstrip Antenna

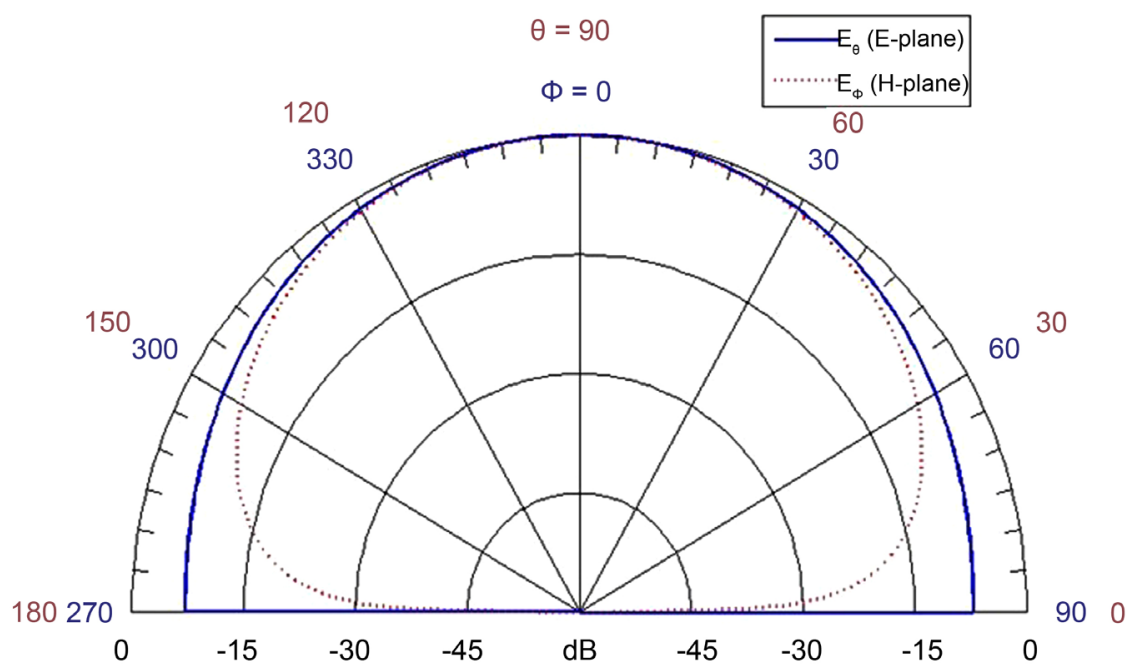

Figure 4. The field patterns of rectangular microstrip antenna.

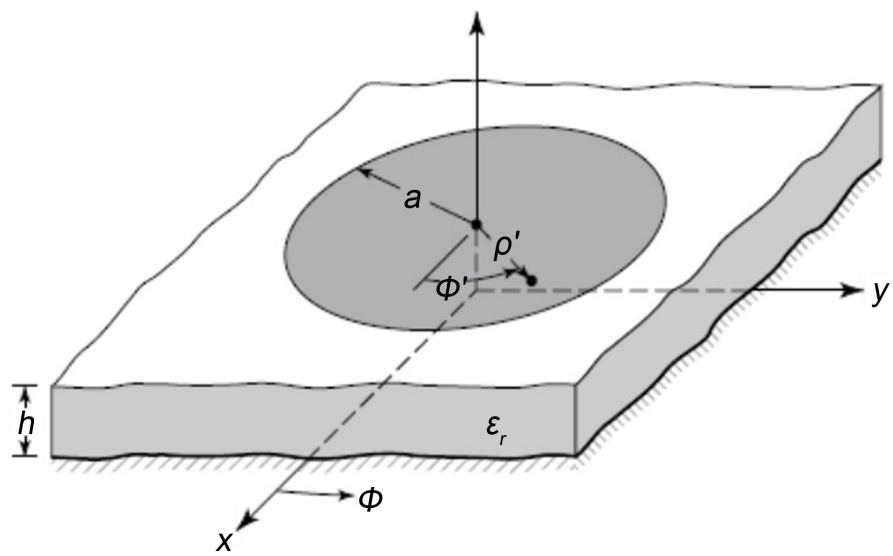

Figure 5. Circular microstrip antenna.

The normalized radiation field for the same in the H-pane $\left(\varphi=0^{\circ}, 0^{\circ} \leq \theta \leq 180^{\circ}\right)$ is given

by

$$
E_{\phi}=J_{0}\left[\sin (\theta)\left(k_{0} a_{e}\right)\right] \cos (\theta)+J_{2}\left[\sin (\theta)\left(k_{0} a_{e}\right)\right] \cos (\theta)
$$


Table 1. Rectangular microstrip design parameter.

\begin{tabular}{cc}
\hline Parameter & Values \\
\hline Length, L & $0.9 \mathrm{~cm}$ \\
Width, W & $1.20 \mathrm{~cm}$ \\
Substrate height, h & $0.16 \mathrm{~cm}$ \\
Feed lenegth, $y_{0}$ & $0.313 \mathrm{~cm}$ \\
Dielectric constant, $\boldsymbol{\varepsilon}_{r}$ & 2.2 \\
Resonant Frequency, $f_{0}$ & $10 \mathrm{GHz}$ \\
\hline
\end{tabular}

where $J_{0}(x)$ is the zeroth order Bessel function, $J_{2}(x)$ is the second order Bessel function, $a_{e}$ is the effective radius. In order to compare the field patterns for circular microstrip antenna with those of rectangular microstrip antenna we maintain the same metallic surface area. We also maintain the same substrate height. Plots of the E-plane and H-plane field patterns for the circular microstrip antenna of same area are shown in Figure 6. The other parameters used to produce the field patterns are as follows: effective radius $a_{e}=0.5985 \mathrm{~cm}$, the E-plane $\mathrm{HPBW}=92^{\circ}$ and the H-plane HPBW $=78^{\circ}$, the directivity is 5.5272 , the input resistance at feed point is $228 \Omega$.

The directivity of circular microstrip and rectangular microstrip antennas are illustrated in Figure 7 and Figure 8. It is shown in Figure 7 that directivity variations for both investigated antennas are not much different when different dielectric materials are used. But, the directivities vary with respect to the height of dielectric material as shown in Figure 8. This figure depicts that the circular microstrip antennas achieve high directivity compared to its rectangular counterpart. Hence, we consider circular microstrip antenna for the rest of the work presented in this paper.

\section{Optimal Smart Antenna Algorithms}

Smart antenna system is composed of a collection of two or more antenna elements called antenna array. These antenna elements work together to establish a unique radiation pattern. A functional block diagram of a smart antenna system is shown in Figure 9. Adaptive algorithm is the main functional component of a smart antenna system. Smart antenna system can locate and track desired signal called signal of interest (SOI) and also signal of no interest (SNOI) by adjusting the antenna weights. By using adaptive algorithms, the smart antenna system can dynamically adjust radiation of the antenna elements so that the resultant radiation is directed along the SOI and it can suppress the radiation patterns along the direction of SNOIs. In order to track the radiation pattern direction of arrival (DOA) algorithm is used. The DOA algorithm helps the antenna elements to set the desired excitation weights in terms of magnitude and phase so that the radiation can be directed in a desired direction.

In smart antenna system the antenna elements may be arranged in many different ways. Most common arrangements are linear, rectangular, and circular as shown in Figure 10. 


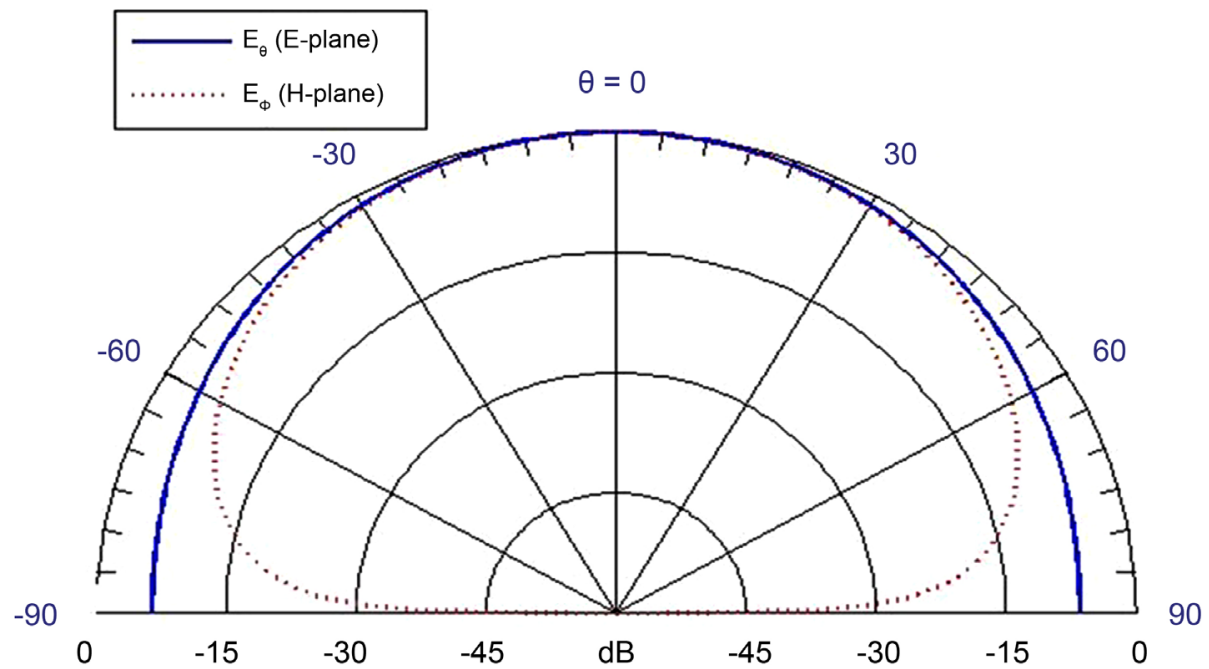

Figure 6. The field patterns of circular microstrip antenna.

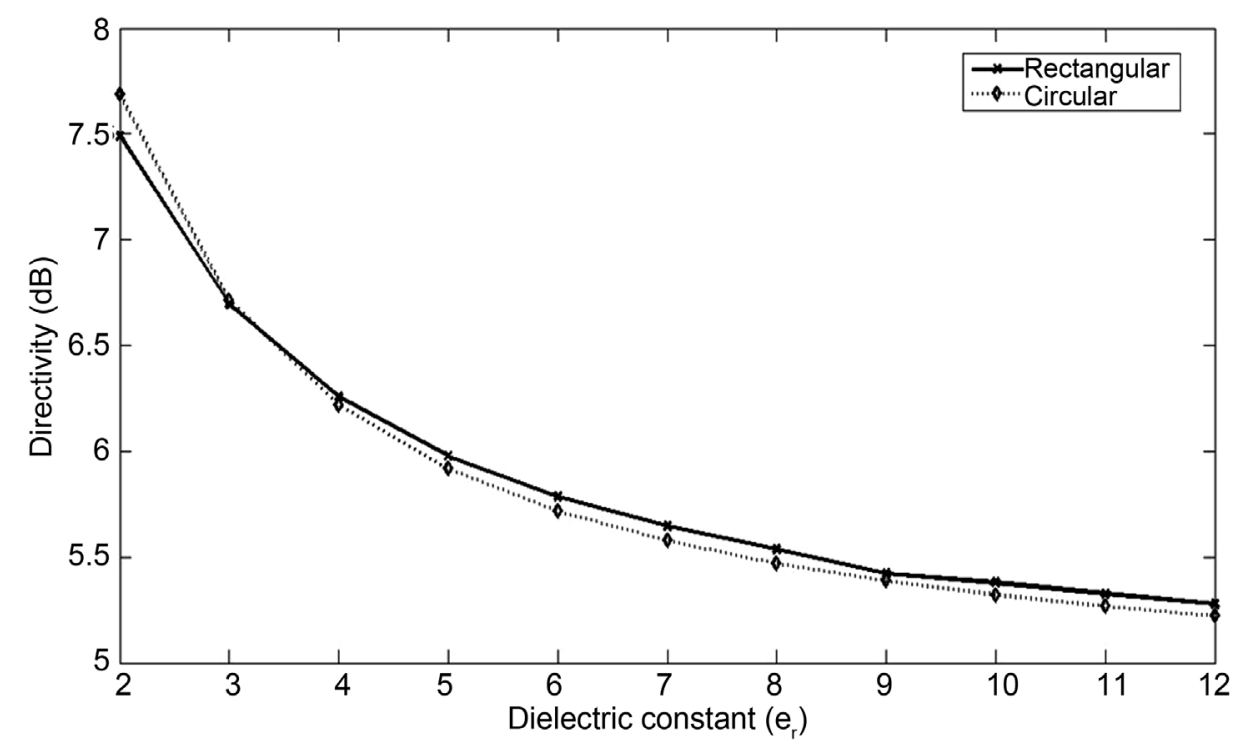

Figure 7. Variation of directivity with respect to dielectric constant.

These arrangements are associated with different level of complexity in design and implementation. A number of investigations have been carried out in order to determine a suitable arrangement for ad hoc network. Among these antenna array arrangements linear array is the simplest one and we used it for this investigation. In linear arrangement all antenna array elements are aligned along a straight line. We assume that antenna elements are equally spaced by a distance d. We also assume that the far-field condition is satisfied (i.e., $r \gg d$ ).

Two different types of beam forming algorithms have been presented in the literatures namely fixed beam forming and adaptive beam forming. Maximum Signal-to-Interference Ratio, Minimum Mean Square Error, Maximum likelihood, and Minimum Variance algorithms are categorized as fixed beam forming 


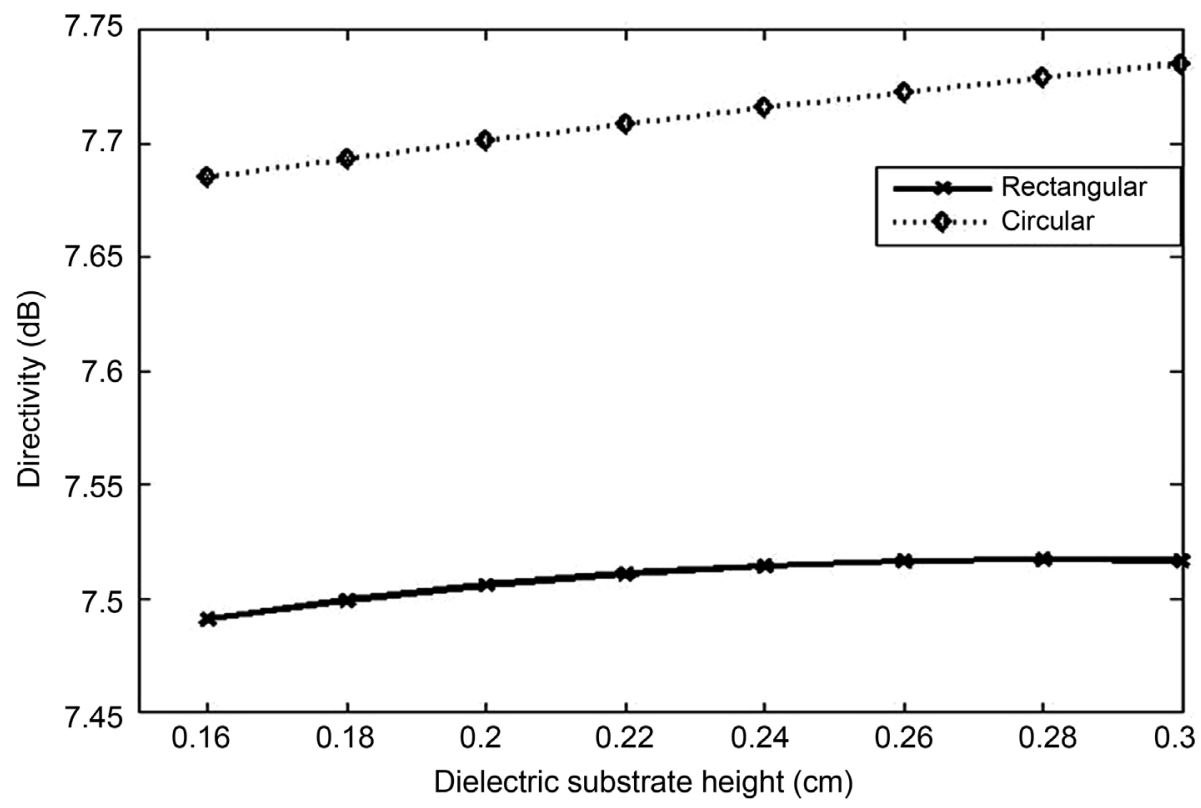

Figure 8. Variation of directivity with respect to dielectric substrate height.

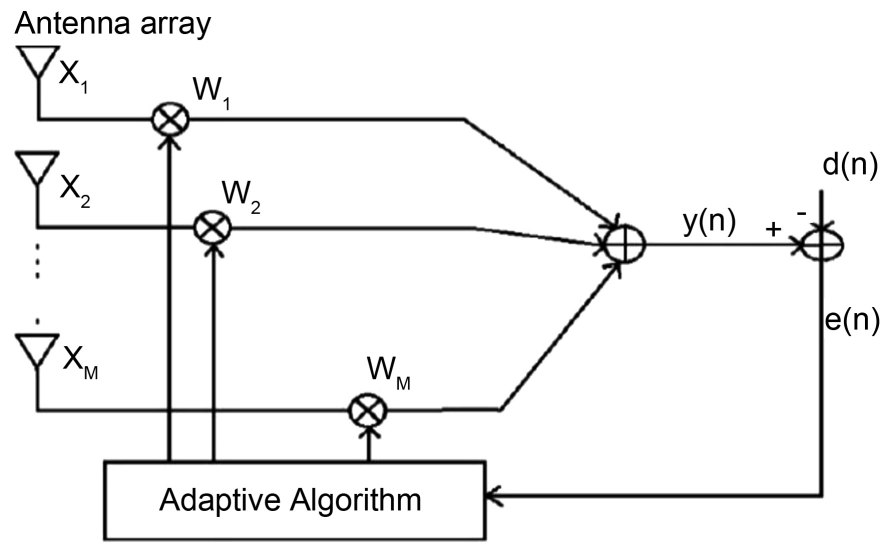

Figure 9. Smart antenna system.

algorithms. These algorithms are suitable for static networks because of their fixed beam forming characteristic. In these networks the signal arrival angle is fixed. Once array weights are determined by the above mentioned algorithm they do not change over the time.

Usually, the ad hoc network has dynamic topology. The mobile nodes can move at any time in any direction. Hence, adaptive beam forming algorithms are considered suitable for such network. There have been several adaptive algorithms proposed in the literatures that are considered suitable for network of dynamic topology. Among them Least Mean Square (LMS), Sample Matrix Inversion (SMI), Recursive Least Square (RLS), Constant Modulus (CM), Least Square Constant Modulus (LSCM), Conjugate Gradient Method (CGM), and Spreading Sequence Array Weights (SSAW) are worthwhile to mention here [14]. These algorithms widely vary in terms of design complexity and computational efficiency. An investigation of all these algorithms is beyond the scope of 


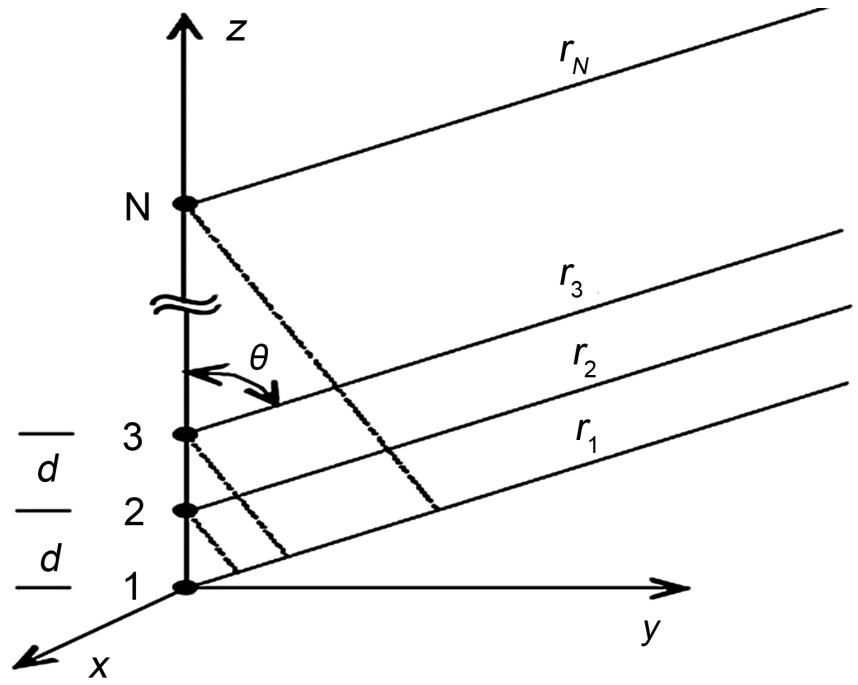

(a)

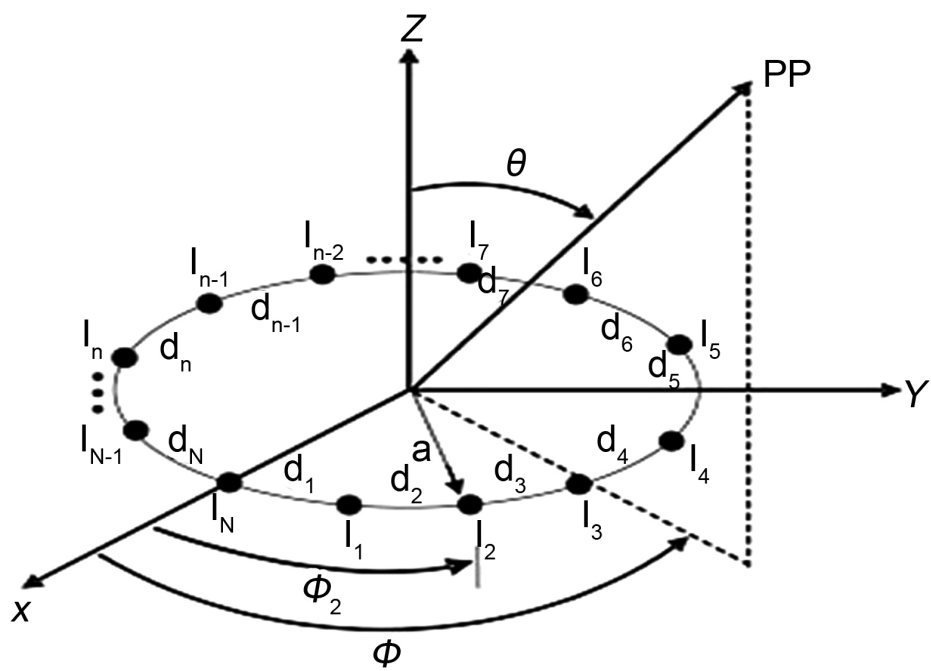

(b)

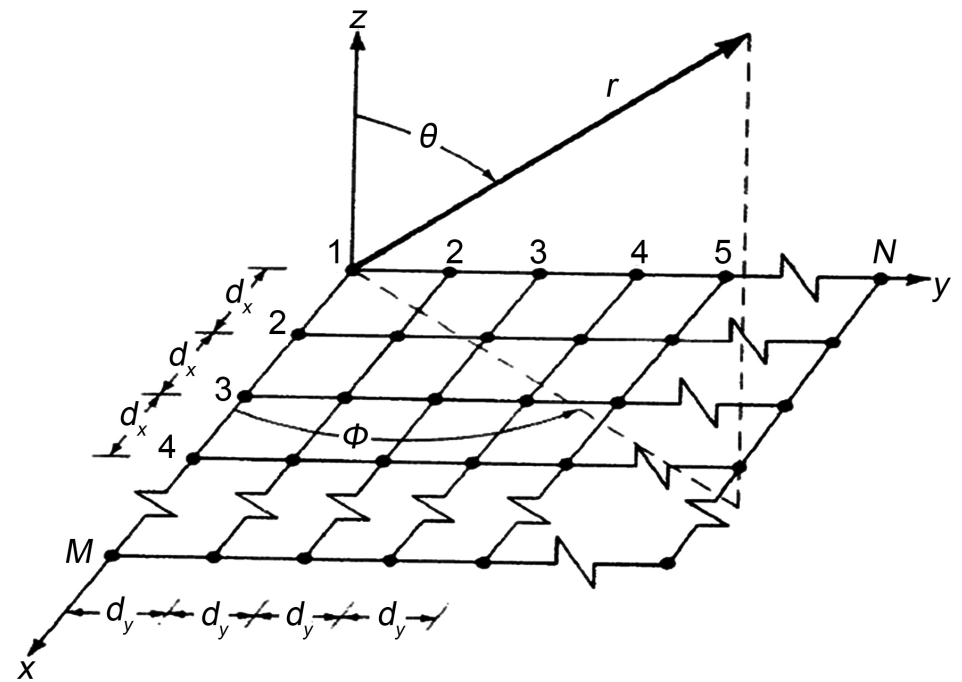

(c)

Figure 10. Antenna arrangements for ad hoc networks. (a) Linear; (b) Circular; (c) Planar. 
this work. In this work we choose some popular algorithms namely LMS algorithm, Sample Matrix Inversion (SMI), Recursive Least Square (RLS), Constant Modulus Algorithm (CMA) and Least Square Constant Modulus Algorithm (LCMA).

The LMS algorithm, proposed in [15], is a gradient based algorithm. It is assumed in this algorithm that there exists a quadratic performance surface. The performance surface is in the shape of elliptic paraboloid having one minimum. A gradient algorithm has been used to establish the minimum condition. An iterative technique called the method of steepest descent has been used in order to determine the gradient of the cost function. By using the algorithm, the weights of the antenna elements are determined by using LMS method introduced in [16] [17].

In order to design smart antenna system for ad hoc network using LMS algorithm we use the MATLAB program provided in [14]. Since the mobile nodes in ad hoc networks have limited processing power, we would like to choose a minimum number of antenna elements to achieve a desired radiation pattern. The antenna weights are approximated by

$$
w(k+1)=w(k)-\frac{1}{2} \mu(\nabla w) J[w(k)]
$$

where $\mu$ is the step-size, $\nabla w$ is the gradient of the performance surface, and $J[w(k)]$ is the cost function. Determining the step size is important for this algorithm and it affects the convergence of the LMS algorithm. If the step size is too small, the convergence of the algorithm will be slow and the adaptive array faces problem to determine the angle of signal arrival. If the step size is too large, the LMS algorithm overshoots and it becomes difficult to achieve optimum weights for the antenna array. It has been shown in [15] that the most suitable value of the step size is set by

$$
0 \leq \mu \leq \frac{1}{2 \lambda_{\max }}
$$

where $\lambda_{\max }$ is the largest value of the correlation matrix of signal vector. In this work we choose $\mu=2 \lambda_{\text {max }}$.

The Sample Matrix Inversion (SMI) is another popular algorithm. This is an improved version of LMS algorithm. The SMI algorithm has been introduced in order to overcome the limitation of LMS algorithm, which requires many iterations before convergence. On the other hand, the SMI algorithm converges quickly [15] [17]. Hence, it is considered more suitable when the signal characteristics are rapidly changing. This type of rapid signal change is very common in ad hoc network environment. The sample matrix used in this algorithm is a time average estimate of the array correlation matrix using $K$-time samples and is given by

$$
\hat{R}_{x x}=\frac{1}{K} \sum_{i=1}^{K} \boldsymbol{x}(i) \boldsymbol{x}^{H}(i)
$$

The correlation vector $r$ can be estimated by 


$$
\hat{r}=\frac{1}{K} \sum_{i=1}^{K} d^{*}(i) x(i)
$$

where $d(k)$ is the desired signal and $x(k)$ is the arriving signals. The weights for the array antenna elements are given by

$$
w(k)=R_{x x}^{-1} \boldsymbol{r}(k)
$$

Although the SMI algorithm is faster than the LMS algorithm, the computational burden is one of the major drawbacks of the SMI algorithm. In order to overcome this, the Recursive Least Square (RLS) algorithm has been introduced. In RLS algorithm the required correlation matrix and the correlation vectors are calculated recursively. In the LMS algorithm more emphasis is given on the recent data samples and less emphasis is given on the past data samples. In this algorithm the sample matrix is given by

$$
\widehat{R}_{x x}(k)=\sum_{i=1}^{K} \alpha^{k-1} \boldsymbol{x}(i) \boldsymbol{x}^{H}(i)
$$

The correlation vector $r$ can be estimated by

$$
\hat{r}=\sum_{i=1}^{K} \alpha^{k-1} d^{*}(i) x(i)
$$

where $\alpha$ is the weight factor. The advantage of the RLS algorithm over SMI is that it is no longer necessary to invert a large correlation matrix. Moreover, the RLS algorithm converges much more quickly than the LMS algorithm.

In many wireless systems the transmitted signal has constant amplitude. This signal is usually affected by multipath propagation that causes signal amplitude to change. Moreover, the constant amplitude property is also affected when the channel is frequency selective. There have been proposals to restore this amplitude variation by using equalization. Some of these equalization algorithms (also called blind equalization) can found [18]. A cost function called dispersion function has been defined in [18] as

$$
J(k)=E\left[\left(|y(k)|^{p}-R_{p}\right)^{q}\right]
$$

where $p$ is the order of the cost function, $q$ is a positive integer, $R_{p}$ is defined as

$$
R_{p}=\frac{E\left[|s(k)|^{2 p}\right]}{E\left[|s(k)|^{p}\right]}
$$

Assuming $p=1$, the antenna weights become

$$
\boldsymbol{w}(k+1)=\boldsymbol{w}(k)+\mu\left(1-\frac{1}{|y(k)|}\right) y^{*}(k) \boldsymbol{x}(k)
$$

In this work we set $p=1$. We assumed there exists one multipath signal in addition to the direct path. The direct path arrives at $0^{\circ}$ and the interfering signal arrives at $-30^{\circ}$.

One of the limitations of CMA algorithm is its slow convergence time. The slow convergence of CMA algorithm limits the applicability of this algorithm in 
ad hoc network environment. Dynamic topology and hostile channel condition of ad hoc network demand a faster algorithm that converges very quickly. The algorithm proposed in [19] is a faster version of CMA algorithm. This algorithm is called Least Square Constant Modulus (LSCM) algorithm. This algorithm is based on autoregressive estimation based on a least square minimization. In this algorithm the cost function is defined as

$$
J(w)=i=\sum_{i=1}^{K}|y(k)|-\left.1\right|^{2}
$$

where $y(k)$ is the array output defined by $y(k)=\boldsymbol{w}^{H} x(k)$. In this algorithm the initial value of the weights are assumed to be zero and the algorithm iterates through $n$ values until it converges with the array weights. It is discovered that the LSCM algorithm is much faster than simple CMA algorithm and it converges with few iterations.

In order to investigate the performances of the above mentioned smart antenna algorithms namely LMS, SMI, RLS, CMA and LSCMA we design a linear antenna array consisting of 8-elements. The antenna elements are separated by a distance of $\lambda / 2$. The desired signal is arrived at $\theta=0^{\circ}$ and the interfering signal is arrived at $\theta=-30^{\circ}$. We assume the desired signal is purely sinusoidal with period $1 \mathrm{mS}$. The radiation patterns for these algorithms are illustrated in Figure 11. The antenna array is operated in a noisy environment. We assume the noise is Gaussian with mean 0 and standard deviation 1. It is depicted in the figure that all the investigated algorithms generate a maximum radiation patter in the desired direction (i.e., $\theta=0^{\circ}$ ). It is also depicted that all the algorithms generate null at the interfering signal (i.e., $\theta=-30^{\circ}$ ) except CMA algorithm. The CMA algorithm only attenuates the signal, but it fails to nullify the signal. In terms of side lobe amplitude, the LMS and LSCMA algorithm performs better compared to other three algorithms. The RLS algorithm produces maximum side lobe. The side lobes attenuate very quickly except SMI algorithm. The side lobe reduces to 0.1 for the SMI algorithm, but the same increases to 0.4 again. Although the LMS and LSCMA show similar performance in terms of side lobe minimization, the LSCMA should be used in the ad hoc networks because of its rapid convergence property compared to its LMS counterpart.

In order to investigate the performances of investigated algorithms under varying noise condition we conduct the similar experiment as mentioned above. But, we set the noise mean to 2 and standard deviation 1 . The generated radiation patterns by the algorithms are shown in Figure 12. It is depicted in the figure that all smart antenna algorithms except RLS produce maximum radiation along the desired direction (i.e., $\theta=0^{\circ}$ ). In terms of side lobe attenuation CMA algorithm outperforms other algorithms. The performance of LSCMA is also very much similar to CMA algorithms. But, CMA outperforms LSCMA.

\section{Conclusion}

In this paper, smart antenna design strategy for ad hoc networks has been presented. Since mobile nodes in ad hoc network have very limited processing 


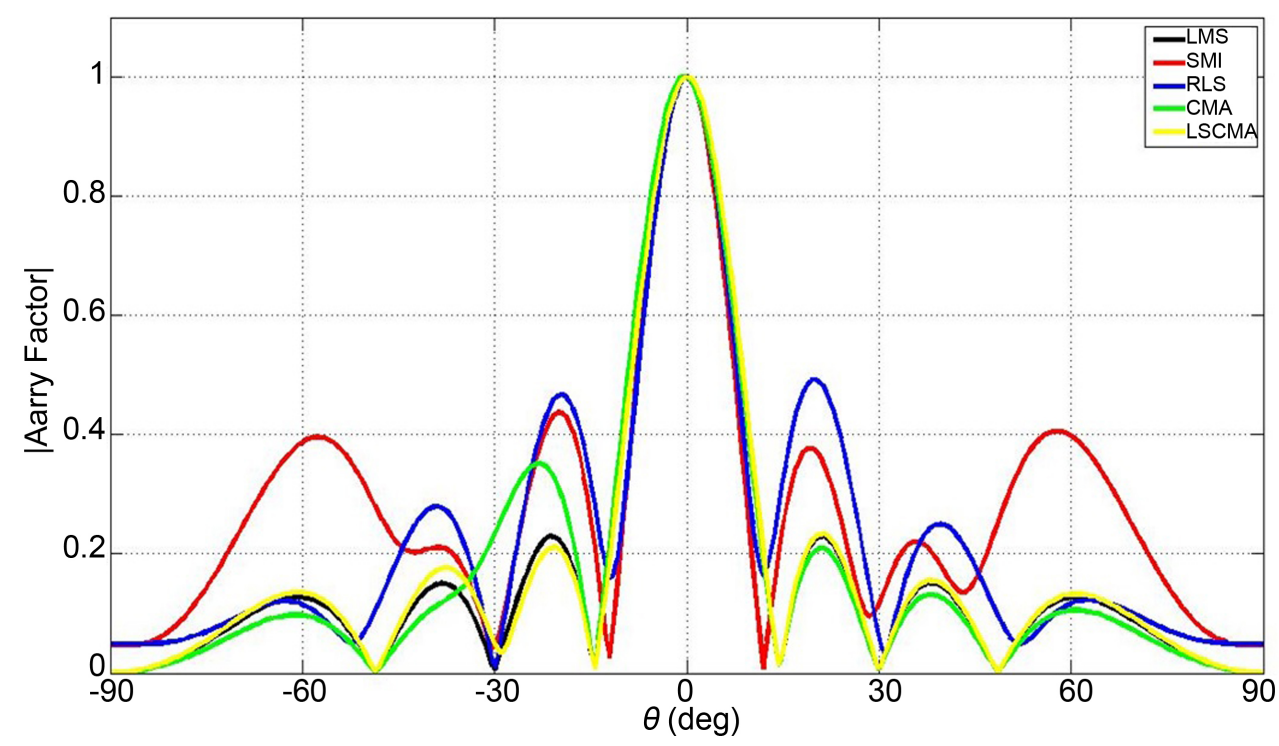

Figure 11. Comparison with noise mean 0 and standard deviation 1.

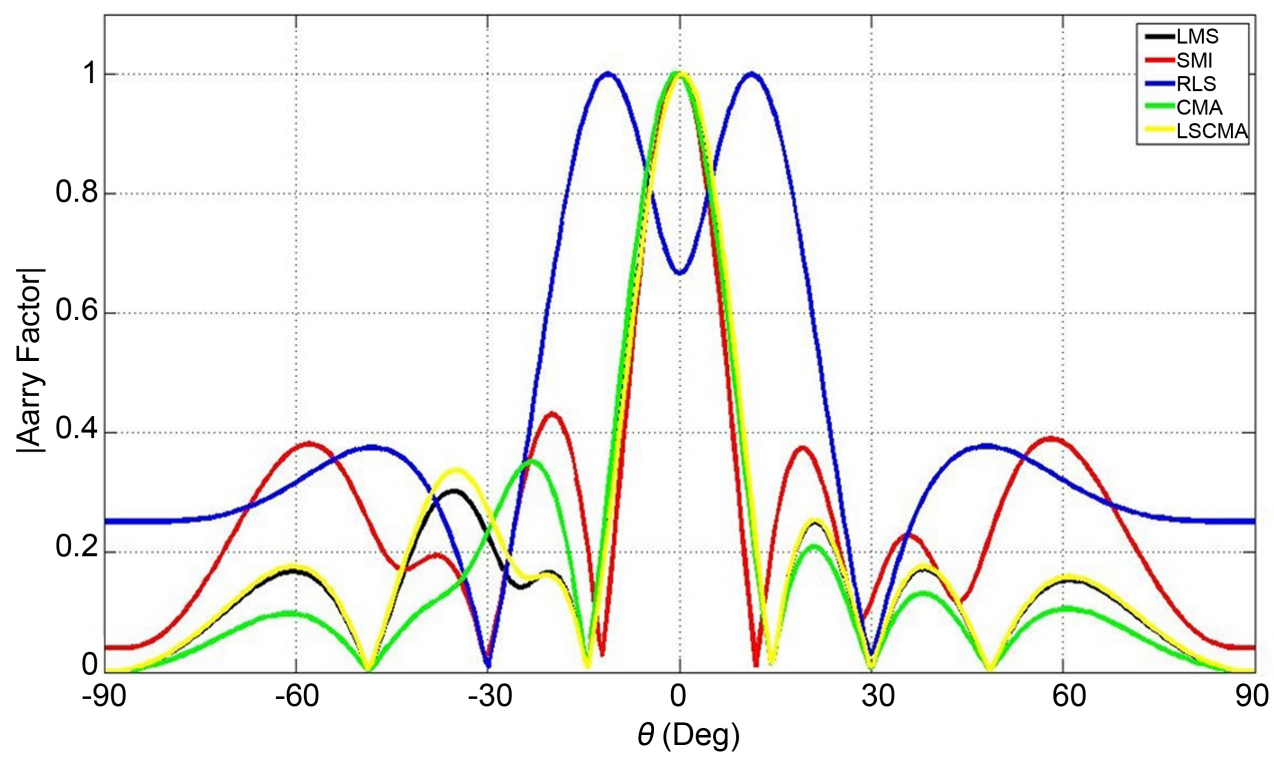

Figure 12. Comparison with noise mean 2 and standard deviation 1.

power, smart antenna should be very simple in architecture. Smart antenna also should consist of limited number of antenna elements. In addition, a suitable antenna element will be chosen. In this paper it is shown that circular microstrip antenna is a good candidate for ad hoc network because of its higher directivity compared to its rectangular counterpart. Once the circular microstrip antenna is chosen as an antenna element, we show that linear antenna arrangement is suitable for ad hoc network because of its simplicity in design. It is also important to find a suitable smart antenna algorithm for ad hoc networks. In this paper we investigated five popular smart antenna algorithms. We simulated and compared the performances of these algorithms. The simulation results show that the LSCMA algorithm is the best candidate for ad hoc network under a low 
noise condition. But, CMA algorithm outperforms LSCMA algorithm under noisier conditions.

\section{References}

[1] Goldsmith, A.J. and Wicker, S.B. (2002) Design Challenges for Energy-Constrained Ad Hoc Wireless Network. IEEE Wireless Communication, 9, 8-27. https://doi.org/10.1109/MWC.2002.1028874

[2] Bellofiore, S., Foutz, J., Govinfarajula, R., Bachceci, I., Balanis, C.A., Spanais, A.S., Capone, J.M. and Duman, T.M. (2002) Smart Antenna System Analysis, Integration, and Performance for Mobile Ad Hoc Networks (MANETs). IEEE Transaction on Antennas and Propagation, 50, 571-581. https://doi.org/10.1109/TAP.2002.1011222

[3] Radhakrishnan, R., Lai, D., Caffery, J. and Agrawal, D.P. (2002) Performance Comparison of Smart Antenna Techniques for Spatial Multiplexing in Ad Hoc Networks. Proceedings of the 5 th International Symposium on Wireless Personal Multimedia Communications, 1, 168-171.

[4] Nasipuri, A., Ye, S., You, J. and Hiromoto, R.E. (2000) A MAC Protocol for Mobile Ad Hoc Networks Using Directional Antennas. Proceedings of IEEE Wireless Communication and Networking Conference, Chicago, IL, Vol. 3, 1214-1219.

[5] Takai, M., Martin, J., Bagrodia, R. and Ren, A. (2002) Directional Virtual Carrier Sensing for Directional Antennas in Mobile Ad Hoc Networks. Proceedings of the 3 rd ACM International Symposium on Mobile Ad Hoc Networking \& Computing, Lausanne, June 2002, 183-193. https://doi.org/10.1145/513800.513823

[6] Roychoudhury, R., Yang, X., Ramanathan, R. and Vaidya, N. (2002) Medium Access Controlin Ad Hoc Networks Using Directional Antenna. Proceedings of the 8 th Annual International Conference on Mobile Computing and Networking, Atlanta, Georgia, 23-28 September 2002, 59-70.

[7] Bao, L. and Garcia-Luna-Aceves, J.J. (2002) Transmission Scheduling in Ad Hoc Networks with Directional Antenna. Proceedings of the 8th Annual International Conference on Mobile Computing and Networking, Atlanta, Georgia, 23-26 September 2002, $11 \mathrm{p}$.

[8] Spyropoulos, A. and Raghabendra, C.S. (2003) Capacity Bounds for Ad hoc Network using Directional Antennas. Proceedings of the IEEE International Conference on Communication, Anchorage, Alaska, May 2003, 348-352.

[9] Ramanthan, R. (2001) On the Performance of Beamforming Antennas in Ad Hoc Networks. Proceedings of the ACM Symposium on Mobile Ad Hoc Networking \& Computing, Long Beach, CA, 4-5 October 2001, 95-105. https://doi.org/10.1145/501416.501430

[10] Spyropoulos, A. and Raghavendra, C.S. (2003) Energy Efficient Communications in Ad Hoc Networks Using Directional Antennas. Proceedings of the 22nd Annual Joint Conference of the IEEE Computer and Communications Societies, San Francisco, CA, March-April, 2003, Vol. 1, 220-228.

[11] Nasipuri, A., Li, K. and Sappidi, U.R. (2002) Power Consumption and Throughput in Mobile Ad Hoc Networks Using Directional Antennas. Proceedings of the 11 th Conference on Computer Communication and Networks, Miami, FL, October 2002, 620-626.

[12] Winters, J.H. (2006) Smart Antenna Techniques and Their Applications to Wireless Ad Hoc Networks. IEEE Wireless Communication, 13, 77-83.

https://doi.org/10.1109/MWC.2006.1678168 
[13] Belanis, C.A. (2005) Antenna Theory: Design and Analysis. 3rd Edition, John Wiley and Sons, NJ, 826-837.

[14] Gross, F. (2005) Smart Antennas for Wireless Communications with Matlab. 2nd Edition, McGraw Hill, New York, 227-251.

[15] Monzingo, R. and Miller, T. (1980) Introduction to Adaptive Arrays. Wiley Interscience, John Wiley and Sons, New York.

[16] Widrow, B., Mantey, P., Griffith, L., et al. (1967) Adaptive Antenna System. Proceedings of IEEE, 55, 2143-2159.

[17] Godara, L. (2004) Smart Antennas. CRC Press, Boca Raton, FL. https://doi.org/10.1201/9780203496770

[18] Godara, D.N. (1980) Self-Recovering Equalization and Carrier Tracking in Two Dimensional Data Communication Systems. IEEE Transactions on Communications, 28, 1867-1875.

[19] Rong, Z. (1996) Simulation of Adaptive Algorithm for CDMA Systems. Master's Thesis, Mobile and Portable Radio Research Group, Virginia Tech, Blacksburg, VA.

\section{Submit or recommend next manuscript to SCIRP and we will provide best} service for you:

Accepting pre-submission inquiries through Email, Facebook, LinkedIn, Twitter, etc. A wide selection of journals (inclusive of 9 subjects, more than 200 journals)

Providing 24-hour high-quality service

User-friendly online submission system

Fair and swift peer-review system

Efficient typesetting and proofreading procedure

Display of the result of downloads and visits, as well as the number of cited articles

Maximum dissemination of your research work

Submit your manuscript at: http://papersubmission.scirp.org/

Or contact wet@scirp.org 